\title{
HIGHLIGHTS
}

MULTIPLE SCLEROSIS

\section{Vascular angioplasty for MS?}

Multiple sclerosis (MS) is a degenerative motor disease, commonly thought to be caused by an autoimmune reaction. Now, Paolo Zamboni, a vascular surgeon in Italy, has suggested that constriction in cerebrospinal blood vessels draining the brain may lead to MS, after he identified and treated blockages here in a group of patients with this condition. These results suggest an alternative causal model for MS, possibly leading to new treatment approaches.

\section{4 ...blockages in the cerebrospinal blood vessels draining the brain may lead to MS... 77}

Chronic cerebrospinal venous insufficiency (CCSVI) is a vascular condition characterized by blockages in the vessels responsible for extracranial drainage. Zamboni noted that several histological markers of CSSVI are often found in patients with MS. He wondered, therefore, whether CSSVI might cause MS, and whether treatment with a common vascular surgical procedure would be feasible, safe, and able to relieve MS symptoms.

65 patients with MS aged 18-65 years with evidence of abnormal extracranial cerebral venous outflow were selected for the study. The patients were screened for physical and hemodynamic indicators of CCSVI and categorized by MS severity. Selective venography of vessels comprising the cerebral venous outflows was then used to detect the size and nature of blockages.

Zamboni et al. performed percutaneous transluminal angioplasty (PTA) - a conservative approach in stenosis treatment-to treat the blockages. The researchers found significantly reduced venous pressure after treatment, and $50-96 \%$ patency rates at 18 month follow-up. Neurological measures showed improvements in MS disease severity and quality of life measures, as well as reduced relapse rates. The extent of this effect was inversely proportional to disease severity, with those with less-severe MS at baseline showing the most improvement.

These findings suggest that PTA can effectively and safely reduce vascular constriction and successfully treat MS symptoms. These results cannot be broadly generalized, however, as the data are preliminary, and the study was not designed for treatment evaluation, with no control group being tested.

It also remains unclear how Zamboni's theory can be reconciled with the autoimmune model, which is supported by a robust body of evidence. $\mathrm{He}$ acknowledges "it may well be that both CCSVI and externally driven CNS autoimmunity are important contributors to MS."

Since this study, preliminary findings from a team in Buffalo, USA have indicated a $55 \%$ prevalence of venous insufficiency in patients with

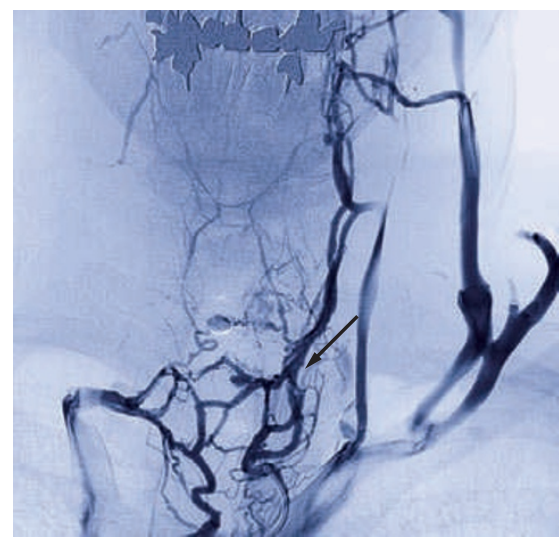

Congenital hypoplasia of the left internal jugular vein, with activation of cervical collaterals vein demonstrated by catheter angiography. Image courtesy of Dr R. Galeotti.

MS, compared with $25 \%$ in controls, supporting the presence of an association between these conditions. More data are needed, however, to elucidate the role of vascular constriction in MS. Mark Haacke, Director of the MRI Institute for Biomedical Research in Detroit, believes that this information could be collected quickly and easily from MS patients by "adding an extra 30-40 min to scans to determine if they have CCSVI," in parallel with additional controlled studies.

Zamboni plans to continue his work along three lines of investigation: first, "to integrate the genetics of CCSVI (and) the genetics of MS," second "to investigate the impact of brain pathophysiology of CCSVI," and third "to seek more therapeutic evidence."

\section{Eleanor Beal}

Original article Zamboni, P. et al. A prospective open-labe study of endovascular treatment of chronic cerebrospinal venous insufficiency. J. Vasc. Surg. 50, 1348-1358 (2009) 